\title{
Boundedness of Fractional Integral with Variable Kernel and Their Commutators on Variable Exponent Herz Spaces
}

\author{
Afif Abdalmonem 1,2, Omer Abdalrhman 1,3, Shuangping Tao ${ }^{{ }^{*}}$ \\ ${ }^{1}$ College of Mathematics and Statistics, Northwest Normal University, Lanzhou, China \\ ${ }^{2}$ Faculty of Science, University of Dalanj, Dalanj, Sudan \\ ${ }^{3}$ College of Education, Shendi University, Shendi, Sudan \\ Email: afeefy86@gmail.com, humoora@gmail.com, taosp@nwnu.edu.cn
}

Received 25 April 2016; accepted 26 June 2016; published 29 June 2016

Copyright (C) 2016 by authors and Scientific Research Publishing Inc.

This work is licensed under the Creative Commons Attribution International License (CC BY).

http://creativecommons.org/licenses/by/4.0/

(c) (i) Open Access

\section{Abstract}

In this paper, we study the boundedness of the fractional integral operator and their commutator on Herz spaecs with two variable exponents $p(\cdot), q(\cdot)$. By using the properties of the variable exponents Lebesgue spaces, the boundedness of the fractional integral operator and their commutator generated by Lipschitz function is obtained on those Herz spaces.

\section{Keywords}

Fractional Integral, Variable Kernel, Commutator, Variable Exponent, Lipschitz Space, Herz Spaces

\section{Introduction}

Let $0<\mu<n, \Omega \in L^{\infty}\left(\mathbb{R}^{n}\right) \times L^{r}\left(S^{n-1}\right)(r \geq 1)$ is homogenous of degree zero on $\mathbb{R}^{n}$, $S^{n-1}$ denotes the unit sphere in $\mathbb{R}^{n}$. If

(i) For any $x, z \in \mathbb{R}^{n}$, one has $\Omega(x, \lambda z)=\Omega(x, z)$;

(ii) $\|\Omega\|_{L^{\infty}\left(\mathbb{R}^{n}\right) \times L^{r}\left(s^{n-1}\right)}:=\sup _{x \in \mathbb{R}^{n}}\left(\int_{s^{n-1}}\left|\Omega\left(x, z^{\prime}\right)\right|^{r} \mathrm{~d} \sigma\left(z^{\prime}\right)\right)^{\frac{1}{r}}<\infty$

The fractional integral operator with variable kernel $T_{\Omega, \mu}$ is defined by

${ }^{*}$ Corresponding author.

How to cite this paper: Abdalmonem, A., Abdalrhman, O. and Tao, S.P. (2016) Boundedness of Fractional Integral with Variable Kernel and Their Commutators on Variable Exponent Herz Spaces. Applied Mathematics, 7, 1165-1182.

http://dx.doi.org/10.4236/am.2016.710104 


$$
T_{\Omega, \mu} f(x)=\int_{\mathbb{R}^{n}} \frac{\Omega(x, x-y)}{|x-y|^{n-\mu}} f(y) \mathrm{d} y,
$$

The commutators of the fractional integral is defined by

$$
\left[b^{m}, T_{\Omega, \mu}\right] f(x)=\int_{\mathbb{R}^{n}} \frac{\Omega(x, x-y)}{|x-y|^{n-\mu}}(b(x)-b(y))^{m} f(y) \mathrm{d} y,
$$

When $\mu \equiv 1$, the above integral takes the Cauchy principal value. At this time $\mu \equiv 0, T_{\Omega, \mu}$ is much more close related to the elliptic partial equations of the second order with variable coefficients. Now we need the further assumption for $\Omega(x, z)$. It satisfies

$$
\int_{s^{n-1}} \Omega\left(x, z^{\prime}\right) \mathrm{d} \sigma\left(z^{\prime}\right)=0, \forall x \in \mathbb{R}^{n}
$$

For $r \geq 1$, we say Kernel function $\Omega(x, z)$ satisfies the $L^{r}$-Dini condition, if $\Omega$ meets the conditions (i), (ii) and

$$
\int_{0}^{1} \frac{\omega_{r}(\delta)}{\delta} \mathrm{d} \delta<\infty
$$

where $\omega_{r}(\delta)$ denotes the integral modulus of continuity of order $r$ of $\Omega$ defined by

$$
\omega_{r}(\delta)=\sup _{x \in \mathbb{R}^{n},|\rho|<\delta}\left(\int_{s^{n-1}}\left|\Omega\left(x, \rho z^{\prime}\right)-\Omega\left(x, z^{\prime}\right)\right|^{r} \mathrm{~d} \sigma\left(z^{\prime}\right)\right)^{\frac{1}{r}}
$$

where $\rho$ is the a rotation in $\mathbb{R}^{n}$

$$
|\rho|=\sup _{z^{\prime} \in S^{n-1}}\left|\rho z^{\prime}-z^{\prime}\right|
$$

when $\Omega \equiv 1, T_{\Omega, \mu}$ is the fraction integral operator

$$
T_{\Omega, \mu} f(x)=\int_{\mathbb{R}^{n}} \frac{|f(y)|}{|x-y|^{n-\mu}} \mathrm{d} y .
$$

The corresponding fractional maximal operator with variable kernel is defined by

$$
M_{\Omega, \mu} f(x)=\sup _{r>0} \frac{1}{r^{n-\mu}} \int_{|x-y|<r}|\Omega(x, x-y)| \mathrm{d} y .
$$

We can easily find that when $\Omega \equiv 1 \quad M_{\Omega}, \mu$ is just the fractional maximal operator

$$
M_{\Omega, \mu} f(x)=\sup _{r>0} \frac{1}{r^{n-\mu}} \int_{|x-y|<r}|f(y)| \mathrm{d} y
$$

Especially, in the case $\mu \equiv 0$, the fractional maximal operator reduces the Hardy-Littelewood maximal operator.

Many classical results about the fractional integral operator with variable kernel have been achieved [1]-[5]. In 1971, Muckenhoupt and Wheeden [6] had proved the operator $T_{\Omega, \mu}$ was bounded from $L^{p}$ to $L^{q}$. In 1991, Kováčik and Rákosník [7] introduced variable exponents Lebesgue and Sobolev spaces as a new method for dealing with nonlinear Dirichet boundary value problem. In the last 20 years, more and more researchers have been interested in the theory of the variable exponent function space and its applications [8]-[14]. In 2012, Wu Huiling and Lan Jiacheng [15] proved the bonudedness property of $T_{\Omega, \mu}$ with a rough kernel on variable exponents Lebesgue spaces.

Recently, Wang and Tao [16] introduced the class of Herz spaces with two variable exponents, and also studied the Parameterized Littlewood-Paley operators and their commutators on Herz spaces with variable exponents.

The main purpose of this paper is to discuss the boundedness of the fractional integral with variable kernel $T_{\Omega, \mu}$ and their commutators $\left[b^{m}, T_{\Omega, \mu}\right]$ are bonuded on Herz spaces with two variable exponents or not. 
Throughout this paper $|E|$ denotes the Lebesgue measure, $\chi_{E}$ means he characteristic function of a measurable set $S \subset \mathbb{R}^{n}$. C always means a positive constant independent of the main parameters and may change from one occurrence to another.

\section{Definition of Function Spaces with Variable Exponent}

In this section we define the Lebesgue spaces with variable exponent and Herz spaces with two variable exponent, and also define the mixed Lebesgue sequence spaces.

Let $E$ be a measurable set in $\mathbb{R}^{n}$ with $|E|>0$. We first define the Lebesgue spaces with variable exponent.

Definition 2.1. see [1] Let $p(\cdot): E \rightarrow[1, \infty)$ be a measurable function. The Lebesgue space with variable exponent $L^{p(\cdot)}(E)$ is defined by

$$
L^{p(\cdot)}(E)=\left\{f \text { is measurable }: \int_{E}\left(\frac{|f(x)|}{\eta}\right)^{p(x)} \mathrm{d} x<\infty \text { for some constant } \eta>0\right\}
$$

The space $L_{l o c}^{p(\cdot)}(E)$ is defined by

$$
L_{\text {loc }}^{p(\cdot)}(E)=\left\{f \text { is measurable }: f \in L^{p(\cdot)}(K) \text { for all compact } K \subset E\right\}
$$

The Lebesgue spaces $L^{p(\cdot)}(E)$ is a Banach spaces with the norm defined by

$$
\|f\|_{L^{p(\cdot)}(E)}=\inf \left\{\eta>0: \int_{E}\left(\frac{|f(x)|}{\eta}\right)^{p(x)} \mathrm{d} x \leq 1\right\}
$$

We denote

$$
p_{-}=\operatorname{essinf}\{p(x): x \in E\}, \quad p_{+}=\operatorname{esssup}\{p(x): x \in E\} .
$$

Then $\mathcal{P}(E)$ consists of all $p(\cdot)$ satisfying $p_{-}>1$ and $p_{+}<\infty$.

Let $M$ be the Hardy-Littlewood maximal operator. We denote $\mathfrak{B}(E)$ to be the set of all function $p(\cdot) \in \mathcal{P}(E)$ satisfying the $M$ is bounded on $L^{p(\cdot)}(E)$.

Definition 2.2. see [17] Let $p(\cdot), q(\cdot) \in \mathcal{P}(E)$. The mixed Lebesgue sequence space with variable exponent $I^{q(\cdot)}\left(L^{p(\cdot)}\right)$ is the collection of all sequences $\left\{f_{j}\right\}_{j=0}^{\infty}$ of the measurable functions on $\mathbb{R}^{n}$ such that

$$
\begin{gathered}
\left\|\left\{f_{j}\right\}_{j=0}^{\infty}\right\| \|_{l^{q(\cdot)}\left(L^{p(\cdot)}\right)}=\inf \left\{\eta>0: Q_{I^{q(\cdot)}\left(L^{p(\cdot)}\right)}\left(\left\{\frac{f_{j}}{\mu}\right\}_{j=0}^{\infty}\right) \leq 1\right\}<\infty \\
Q_{l^{q(\cdot)}\left(L^{p(\cdot)}\right)}\left(\left\{f_{j}\right\}_{j=0}^{\infty}\right)=\sum_{j=0}^{\infty} \inf \left\{\mu_{j} ; \int_{R^{n}}\left(\frac{\left|f_{j}(x)\right|}{\mu_{j}^{q(x)}}\right)^{p(x)} \mathrm{d} x \leq 1\right\}
\end{gathered}
$$

Noticing $q_{+}<\infty$, we see that

$$
Q_{I^{q(\cdot)}\left(L^{p(\cdot)}\right)}\left(\left\{f_{j}\right\}_{j=0}^{\infty}\right)=\sum_{j=0}^{\infty}\left\|\left|f_{j}\right|^{q(\cdot)}\right\|_{L^{q(\cdot)}}
$$

Let $B_{k}=\left\{x \in \mathbb{R}^{n}:|x| \leq 2^{k}\right\}, C_{k}=B_{k} \backslash B_{k-1}, \chi_{k}=\chi_{C_{k}}, k \in \mathbb{Z}$

Definition 2.3. see [16] Let $\alpha \in \mathbb{R}, p(\cdot), q(\cdot) \in \mathcal{P}\left(\mathbb{R}^{n}\right)$. The homogeneous Herz space with variable exponent $\dot{K}_{p(\cdot)}^{\alpha, q(\cdot)}\left(\mathbb{R}^{n}\right)$ is defined by

where

$$
\dot{K}_{p(\cdot)}^{\alpha, q(\cdot)}\left(\mathbb{R}^{n}\right)=\left\{f \in L_{\text {Loc }}^{p(\cdot)}\left(\mathbb{R}^{n} \backslash\{0\}\right):\|f\|_{\dot{K}_{p(\cdot)}^{\alpha, q(\cdot)}\left(\mathbb{R}^{n}\right)}<\infty\right\}
$$




$$
\|f\|_{\dot{K}_{p(\cdot)}^{\alpha, q(\cdot)}\left(\mathbb{R}^{n}\right)}=\|\left\{2^{k \alpha}\left|f \chi_{k}\right|_{k=0}^{\infty} \|_{l^{(\cdot)}\left(L^{p(\cdot)}\right)}=\inf \left\{\eta>0: \sum_{k=-\infty}^{\infty}\left\|\left(\frac{2^{k \alpha}\left|f \chi_{k}\right|}{\eta}\right)^{q(\cdot)}\right\|_{\frac{p(\cdot)}{q^{(\cdot)}}} \leq 1\right\}\right.
$$

Remark 2.1. see [16] (1) If $q_{1}(\cdot), q_{2}(\cdot) \in \mathcal{P}\left(\mathbb{R}^{n}\right)$ satisfying $\left(q_{1}\right)_{+} \leq\left(q_{2}\right)_{+}$, then

$$
\dot{K}_{p(\cdot)}^{\alpha, q_{1}(\cdot)}\left(\mathbb{R}^{n}\right) \subset \dot{K}_{p(\cdot)}^{\alpha, q_{2}(\cdot)}\left(\mathbb{R}^{n}\right)
$$

(2) If $q_{1}(\cdot), q_{2}(\cdot) \in \mathcal{P}\left(\mathbb{R}^{n}\right)$ and $\left(q_{1}\right)_{+} \leq\left(q_{2}\right)_{+}$, then $\frac{q_{2}(\cdot)}{q_{1}(\cdot)} \in \mathcal{P}\left(\mathbb{R}^{n}\right)$ and $\frac{q_{2}(\cdot)}{q_{1}(\cdot)} \geq 1$. Thus, by Lemma 3.7 and Remark 2.2, for any $f \in \dot{K}_{p(\cdot)}^{\alpha, q(\cdot)}\left(\mathbb{R}^{n}\right)$, we have

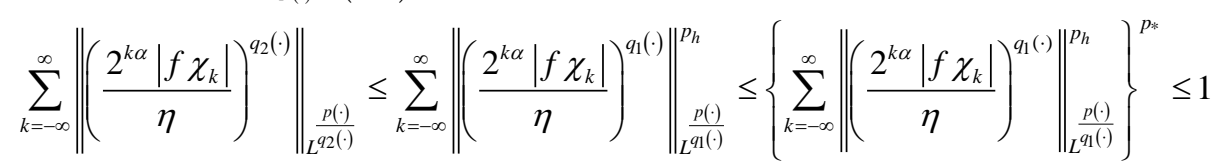

where

$$
\begin{gathered}
p_{h}= \begin{cases}\left(\frac{q_{2}(\cdot)}{q_{1}(\cdot)}\right)_{-}, & \frac{2^{k \alpha}\left|f \chi_{k}\right|}{\eta} \leq 1 \\
\left(\frac{q_{2}(\cdot)}{q_{1}(\cdot)}\right)_{+}, & \frac{2^{k \alpha}\left|f \chi_{k}\right|}{\eta}>1\end{cases} \\
p_{*}= \begin{cases}\min _{h \in \mathbb{N}} p_{h}, & \sum_{h=0}^{\infty} a_{h} \leq 1 \\
\max _{h \in \mathbb{N}} p_{h}, & \sum_{h=0}^{\infty} a_{h}>1\end{cases}
\end{gathered}
$$

This implies that $\dot{K}_{p(\cdot)}^{\alpha, q_{1}(\cdot)}\left(\mathbb{R}^{n}\right) \subset \dot{K}_{p(\cdot)}^{\alpha, q_{2}(\cdot)}\left(\mathbb{R}^{n}\right)$.

Remark 2.2. Let $h \in \mathbb{N}, a_{h} \geq 0,1 \leq p_{h}<\infty$. then

where

$$
\sum_{h=0}^{\infty} a_{h} \leq\left(\sum_{h=0}^{\infty} a_{h}\right)^{p_{*}}
$$

$$
p_{*}= \begin{cases}\min _{h \in \mathbb{N}} p_{h}, & \sum_{h=0}^{\infty} a_{h} \leq 1 \\ \max _{h \in \mathbb{N}} p_{h}, & \sum_{h=0}^{\infty} a_{h}>1\end{cases}
$$

Definition 2.4. see [18] For $0<\beta \leq 1$, the Lipschitz space $\operatorname{Lip}_{\beta}\left(\mathbb{R}^{n}\right)$ is defined by

$$
\operatorname{Lip}_{\beta}\left(\mathbb{R}^{n}\right)=\left\{f:\|f\|_{L i p_{\beta}}=\sup _{x, y \in \mathbb{R}^{n}, x \neq y} \frac{|f(x)-f(y)|}{|x-y|^{\beta}}<\infty\right\}
$$

\section{Properties of Variable Exponent}

In this section we state some properties of variable exponent belonging to the class $\mathfrak{B}\left(\mathbb{R}^{n}\right)$ and $\Omega \in L^{\infty}\left(\mathbb{R}^{n}\right) \times L^{r}\left(S^{n-1}\right)$.

Proposition 3.1. see [1] If $p(\cdot) \in \mathcal{P}\left(\mathbb{R}^{n}\right)$ satisfies

$$
|p(x)-p(y)| \leq \frac{-C}{\log (|x-y|)},|x-y| \leq 1 / 2
$$




$$
|p(x)-p(y)| \leq \frac{C}{\log (e+|x|)},|y| \geq|x|
$$

then, we have $p(\cdot) \in \mathfrak{B}\left(\mathbb{R}^{n}\right)$.

Proposition 3.2. see [15] Suppose that $p_{1}(\cdot) \in \mathfrak{B}\left(\mathbb{R}^{n}\right), \Omega \in L^{\infty}\left(\mathbb{R}^{n}\right) \times L^{r}\left(S^{n-1}\right)$. Let $0<\mu \leq \frac{n}{\left(p_{1}\right)_{+}}$, and define the variable exponent $p_{2}(\cdot)$ by: $\frac{1}{p_{1}(x)}-\frac{1}{p_{2}(x)}=\frac{\mu}{n}$. Then we have that for all $f \in L^{p_{1}(\cdot)}\left(\mathbb{R}^{n}\right)$,

$$
\left\|T_{\Omega, \mu} f\right\|_{L^{p^{2}(\cdot)}\left(\mathbb{R}^{n}\right)} \leq c\|f\|_{L^{p_{1}(\cdot)}\left(\mathbb{R}^{n}\right)}
$$

Proposition 3.3. Suppose that $p_{1}(\cdot) \in \mathfrak{B}\left(\mathbb{R}^{n}\right), \quad b \in \operatorname{Lip}_{\beta}\left(\mathbb{R}^{n}\right), \quad 0<\beta \leq 1, \quad \Omega \in L^{\infty}\left(\mathbb{R}^{n}\right) \times L^{r}\left(S^{n-1}\right)$. Let $0<\mu+m \beta \leq \frac{n}{\left(p_{1}\right)_{+}}$, and define the variable exponent $p_{2}($.$) by: \frac{1}{p_{1}(x)}-\frac{1}{p_{2}(x)}=\frac{\mu+m \beta}{n}$. Then

$$
\left\|\left[b^{m}, T_{\Omega, \mu}\right] f\right\|_{L^{p_{2}(\cdot)}\left(\mathbb{R}^{n}\right)} \leq C\|b\|_{L i p_{\beta}}^{m}\|f\|_{L^{p_{1}(\cdot)}\left(\mathbb{R}^{n}\right)}
$$

\section{Proof}

$$
\begin{aligned}
{\left[b^{m}, T_{\Omega, \mu}\right] f(x) } & =\int_{\mathbb{R}^{n}} \frac{\Omega(x, x-y)}{|x-y|^{n-\mu}}(b(x)-b(y))^{m} f(y) \mathrm{d} y \\
& \leq \int_{\mathbb{R}^{n}} \frac{|\Omega(x, x-y)|}{|x-y|^{n-\mu}}|(b(x)-b(y))|^{m}|f(y)| \mathrm{d} y \\
& \leq C\|b\|_{L i p_{\beta}} \int_{\mathbb{R}^{n}} \frac{|\Omega(x, x-y)|}{|x-y|^{n-\mu-m \beta}}|f(y)| \mathrm{d} y \\
& \leq C\|b\|_{L_{i p_{\beta}}} T_{|\Omega|, \mu+m \beta}(|f|)
\end{aligned}
$$

By Proposition 3.2, we get

$$
\left\|\left[b^{m}, T_{\Omega, \mu}\right] f\right\|_{L^{p^{2}(\cdot)}\left(\mathbb{R}^{n}\right)} \leq C\|b\|_{L_{i p_{\beta}}}\left\|T_{|\Omega|, \mu+m \beta}(|f|)\right\|_{L^{p_{2}(\cdot)}} \leq C\|b\|_{L_{i i_{\beta}}}^{m}\|f\|_{L^{p_{1}(\cdot)}\left(\mathbb{R}^{n}\right)} \cdot
$$

Now, we need recall some lemmas

Lemma 3.1. see [13] Given $p(\cdot): \mathbb{R}^{n} \rightarrow[1, \infty)$ have that for all function $f$ and $g$,

$$
\int_{\mathbb{R}^{n}}|f(x) g(x)| \mathrm{d} x \leq C\|f\|_{L^{(\cdot)}\left(\mathbb{R}^{n}\right)}\|g\|_{L^{p^{\prime} \cdot(\cdot)}\left(\mathbb{R}^{n}\right)}
$$

Lemma 3.2. see [19] Suppose that $0<\mu<n, r>1, \Omega \in L^{\infty}\left(\mathbb{R}^{n}\right) \times L^{r}\left(S^{n-1}\right)$ satisfies the $L^{r}$-Dini condition. If there exists an $0<\alpha_{0}<1 / 2$ such that $|y|<\alpha_{0} R$ then

$$
\left(\int_{R \leq|x|<2 R}\left|\frac{\Omega(x, x-y)}{|x-y|^{n-\mu}}-\frac{\Omega(x, x)}{|x|^{n-\mu}}\right|^{r} \mathrm{~d} x\right)^{\frac{1}{r}} \leq C R^{\left(\frac{n}{r}-n+\mu\right)}\left(\frac{|y|}{R}+\int_{|y| / 2 R}^{|y| / R} \frac{\omega_{r}(\delta)}{\delta} \mathrm{d} \delta\right)
$$

Lemma 3.3. see [20] Suppose that $x \in \mathbb{R}^{n}$, the variable function $\tilde{q}(x)$ is defined by $\frac{1}{p(x)}=\frac{1}{q}+\frac{1}{\tilde{q}(x)}$, then for all measurable function $f$ and $g$, we have

$$
\|f(x) g(x)\|_{L^{p(\cdot)}\left(\mathbb{R}^{n}\right)} \leq C\|g(x)\|_{L^{q}\left(\mathbb{R}^{n}\right)}\|f(x)\|_{L^{\tilde{q}(\cdot)}\left(\mathbb{R}^{n}\right)}
$$

Lemma 3.4. see [21] Suppose that $p(\cdot) \in \mathfrak{B}\left(\mathbb{R}^{n}\right)$ and $0<p^{-} \leq p^{+}<\infty$. 
1) For any cube and $|Q| \leq 2^{n}$, all the $\chi \in Q$, then: $\left\|\chi_{Q}\right\|_{L^{p(\cdot)}} \approx|Q|^{1 / p(x)}$

2) For any cube and $|Q| \geq 1$, then $\left\|\chi_{Q}\right\|_{L^{p(\cdot)}} \approx|Q|^{1 / p_{\infty}}$ where

$$
p_{\infty}=\lim _{x \rightarrow \infty} p(x)
$$

Lemma 3.5. see [22] If $p(\cdot) \in \mathfrak{B}\left(\mathbb{R}^{n}\right)$, then there exist constants $\delta_{1}, \delta_{2}, C>0$ such that for all balls $B$ in $\mathbb{R}^{n}$ and all measurable subset $S \subset R$

$$
\begin{aligned}
& \frac{\left\|\chi_{B}\right\|_{L^{p(\cdot)}}\left(\mathbb{R}^{n}\right)}{\left\|\chi_{S}\right\|_{L^{p(\cdot)}}\left(\mathbb{R}^{n}\right)} \leq C \frac{|B|}{|S|}, \frac{\left\|\chi_{S}\right\|_{L^{p^{i}(\cdot)}\left(\mathbb{R}^{n}\right)}}{\left\|\chi_{B}\right\|_{L^{p^{(}(\cdot)}\left(\mathbb{R}^{n}\right)}} \leq C\left(\frac{|S|}{|B|}\right)^{\delta_{1}}, \\
& \frac{\left\|\chi_{S}\right\|_{L^{p_{1}(\cdot)}\left(\mathbb{R}^{n}\right)}}{\left\|\chi_{B}\right\|_{L^{p_{1}(\cdot)}\left(\mathbb{R}^{n}\right)}} \leq C\left(\frac{|S|}{|B|}\right)^{\delta_{2}}
\end{aligned}
$$

Lemma 3.6. see [13] If $p(\cdot) \in \mathfrak{B}\left(\mathbb{R}^{n}\right)$, there exist a constant $C>0$ such that for any balls $\mathrm{B}$ in $\mathbb{R}^{n}$. we have

$$
\frac{1}{|B|}\left\|\chi_{B}\right\|_{L^{p(\cdot)}\left(\mathbb{R}^{n}\right)}\left\|\chi_{B}\right\|_{L^{p^{\prime}(\cdot)}\left(\mathbb{R}^{n}\right)} \leq C
$$

Lemma 3.7. see [16] Let $p(\cdot), q(\cdot) \in \mathcal{P}\left(\mathbb{R}^{n}\right)$. If $f \in L^{p(\cdot) q(\cdot)}$, then

$$
\min \left(\|f\|_{L^{(\cdot) q(\cdot)}}^{q_{t^{\prime}}},\|f\|_{L^{p(\cdot) q(\cdot)}}^{q_{-}}\right) \leq\left\|\left.f\right|^{q(\cdot)}\right\|_{L^{p(\cdot)}} \leq \max \left(\|f\|_{L^{p(\cdot) q(\cdot)}}^{q_{+}},\|f\|_{L^{p(\cdot) q(\cdot)}}^{q_{-}}\right)
$$

\section{Main Theorems and Their Proof}

Theorem 1. Suppose that $0<\mu<n, \mu-n \delta_{2}<\alpha<n \delta_{1}, \Omega \in L^{\infty}\left(\mathbb{R}^{n}\right) \times L^{r}\left(S^{n-1}\right)\left(r>p_{2}^{+}\right)$,

$q_{1}(\cdot), q_{2}(\cdot) \in \mathcal{P}\left(\mathbb{R}^{n}\right)$ with $\left(q_{2}\right)_{-} \geq\left(q_{1}\right)_{+}$. And let $p_{1}(\cdot) \in \mathfrak{B}\left(\mathbb{R}^{n}\right)$ satisfy $0<\mu \leq \frac{n}{\left(p_{1}\right)_{+}}$and define the variable exponent $p_{2}(x)$ by $\frac{1}{p_{1}(x)}-\frac{1}{p_{2}(x)}=\frac{\mu}{n}$. Then the operators $T_{\Omega, \mu}$ is bounded from $\dot{K}_{p_{1}(\cdot)}^{\alpha, q_{1}(\cdot)}\left(\mathbb{R}^{n}\right)$ to $\dot{K}_{p_{2}(\cdot)}^{\alpha, q_{2}(\cdot)}\left(\mathbb{R}^{n}\right)$.

Theorem 2. Let $b \in \operatorname{Lip}_{\beta}\left(\mathbb{R}^{n}\right), m \in \mathbb{N}$. Suppose that $0<\mu<n,(\mu+m \beta)-n \delta_{2}<\alpha<n \delta_{1}$, $\Omega \in L^{\infty}\left(\mathbb{R}^{n}\right) \times L^{r}\left(S^{n-1}\right)\left(r>p_{2}^{+}\right), q_{1}(\cdot), q_{2}(\cdot) \in \mathcal{P}\left(\mathbb{R}^{n}\right)$ with $\left(q_{2}\right)_{-} \geq\left(q_{1}\right)_{+}$. If $p_{1}(\cdot) \in \mathfrak{B}\left(\mathbb{R}^{n}\right)$ satisfy $0<\mu+m \beta \leq \frac{n}{\left(p_{1}\right)_{+}}$and define the variable exponent $p_{2}(x)$ by $\frac{1}{p_{1}(x)}-\frac{1}{p_{2}(x)}=\frac{\mu+m \beta}{n}$. Then the commutators $\left[b^{m}, T_{\Omega, \mu}\right]$ is bounded from $\dot{K}_{p_{1}(\cdot)}^{\alpha, q_{1}(\cdot)}\left(\mathbb{R}^{n}\right)$ to $\dot{K}_{p_{2}(\cdot)}^{\alpha, q_{2}(\cdot)}\left(\mathbb{R}^{n}\right)$.

\section{Proof of Theorem1:}

Let $f \in \dot{K}_{p_{1}(\cdot)}^{\alpha, q_{1} \cdot(\cdot)}\left(\mathbb{R}^{n}\right)$. We write

$$
f(x)=\sum_{j=-\infty}^{\infty} f(x) \chi_{j}=\sum_{j=-\infty}^{\infty} f_{j}(x)
$$

From definition of $\dot{K}_{p(\cdot)}^{\alpha, q(\cdot)}\left(\mathbb{R}^{n}\right)$

$$
\left\|T_{\Omega, \mu}, f\right\|_{\dot{K}_{p_{2}(\cdot)}^{\alpha, q_{2}(\cdot)\left(\mathbb{R}^{n}\right)}}=\inf \left\{\eta>0: \sum_{k=-\infty}^{\infty}\left\|\left(\frac{2^{k \alpha}\left|T_{\Omega, \mu}(f) \chi_{k}\right|}{\eta}\right)^{q_{2}(\cdot)}\right\|_{\frac{p_{2}(\cdot)}{L_{2(\cdot)}}} \leq 1\right\}
$$

Since 


$$
\begin{aligned}
& \left\|\left(\frac{2^{k \alpha}\left|T_{\Omega, \mu}(f) \chi_{k}\right|}{\eta}\right)^{q_{2}(\cdot)}\right\|_{L_{L}^{p_{2}(\cdot)}} \\
& \leq\left\|\left.\frac{2^{k \alpha}\left|\sum_{j=-\infty}^{\infty} T_{\Omega, \mu}\left(f_{j}\right) \chi_{k}\right|}{\eta_{11}+\eta_{12}+\eta_{13}}\right|^{q_{2}(\cdot)}\right\|_{\frac{p_{2}(\cdot)}{q_{2}(\cdot)}} \leq\left\|\left.\frac{2^{k \alpha}\left|\sum_{j=-\infty}^{k-2} T_{\Omega, \mu}\left(f_{j}\right) \chi_{k}\right|}{\eta_{11}}\right|^{q_{2}(\cdot)}\right\|_{\frac{p_{2}(\cdot)}{q_{2}(\cdot)}} \\
& +\left\|\frac{\left.2^{k \alpha}\left|\sum_{j=k-1}^{k+1} T_{\Omega, \mu}\left(f_{j}\right) \chi_{k}\right|\right|^{q_{2}(\cdot)}}{\eta_{12}}\right\|_{\frac{p_{2}(\cdot)}{q_{2}(\cdot)}}+\left\|\left(\frac{2^{k \alpha}\left|\sum_{j=k+2}^{\infty} T_{\Omega, \mu}\left(f_{j}\right) \chi_{k}\right|}{\eta_{13}}\right)^{q_{2}(\cdot)}\right\|_{\substack{p_{2}(\cdot) \\
L_{2}(\cdot)}}
\end{aligned}
$$

where

$$
\begin{aligned}
& \eta_{11}=\left\|\left\{2^{k \alpha}\left|\sum_{j=-\infty}^{k-2} T_{\Omega, \mu}\left(f_{j}\right) \chi_{k}\right|\right\}_{k=-\infty}^{\infty}\right\| \|_{l^{2}(\cdot)\left(L^{p_{2}(\cdot)}\right)} \\
& \eta_{12}=\left\|\left\{2^{k \alpha}\left|\sum_{j=k-1}^{k+1} T_{\Omega, \mu}\left(f_{j}\right) \chi_{k}\right|\right\}_{k=-\infty}^{\infty}\right\| \|_{q^{q_{2}(\cdot)}\left(L^{p_{2}(\cdot)}\right)} \\
& \eta_{13}=\left\|\left\{2^{k \alpha}\left|\sum_{j=k+2}^{\infty} T_{\Omega, \mu}\left(f_{j}\right) \chi_{k}\right|\right\}_{k=-\infty}^{\infty}\right\| \|_{I^{q_{2}}(\cdot)\left(L^{p_{2}(\cdot)}\right)}
\end{aligned}
$$

And $\eta=\eta_{11}+\eta_{12}+\eta_{13}$, thus

$$
\sum_{k=-\infty}^{\infty}\left\|\left(\frac{2^{k \alpha}\left|T_{\Omega, \mu}\left(f_{j}\right) \chi_{k}\right|}{\eta}\right)^{q_{2}(\cdot)}\right\|_{\frac{p_{2}(\cdot)}{q_{2}(\cdot)}} \leq C
$$

That is

$$
\left\|T_{\Omega, \mu}(f)\right\|_{\dot{K}_{p_{2}(\cdot)}^{\alpha, q_{2}(\cdot)\left(\mathbb{R}^{n}\right)}} \leq C \eta=C\left[\eta_{11}+\eta_{12}+\eta_{13}\right] .
$$

This implies only to prove $\eta_{11}, \eta_{12}, \eta_{13} \leq C\|f\|_{\dot{K}_{p_{1}(\cdot)}^{\alpha, q_{1}(\cdot)}\left(\mathbb{R}^{n}\right)}$. Denote $\eta_{1}=\|f\|_{\dot{K}_{p_{1}(\cdot)}^{\alpha, q_{1}(\cdot)}\left(\mathbb{R}^{n}\right)}$

Now we consider $\eta_{12}$. Applying Lemma 3.7

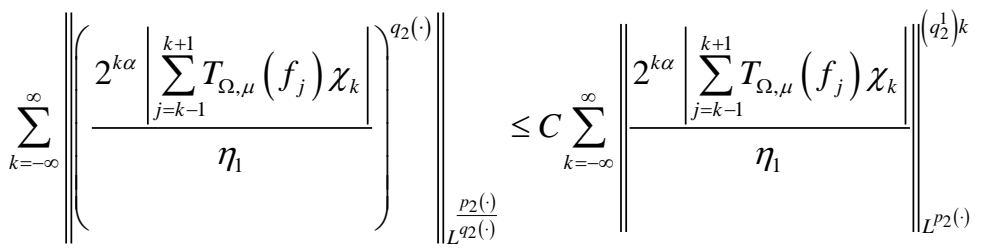

$$
\begin{aligned}
& \leq C \sum_{k=-\infty}^{\infty}\left(\sum_{j=k-1}^{k+1}\left\|\frac{2^{k \alpha}\left|T_{\Omega, \mu}\left(f_{j}\right) \chi_{k}\right|}{\eta_{1}}\right\|_{L^{p_{2}(\cdot)}}\right)^{\left(q_{2}^{1}\right)^{k}}
\end{aligned}
$$


where

$$
\left(q_{2}^{1}\right) k=\left\{\begin{array}{l|l|}
\left(q_{2}\right)_{-}, & \left\|\left(\frac{2^{k \alpha}\left|\sum_{j=k-1}^{k+1} T_{\Omega, \mu}\left(f_{j}\right) \chi_{k}\right|}{\eta_{1}}\right)^{q_{2}(\cdot)}\right\|_{\frac{p_{2}(\cdot)}{q_{2}(\cdot)}} \leq 1 \\
\left(q_{2}\right)_{+}, & \left.\| \frac{2^{k \alpha}\left|\sum_{j=k-1}^{k+1} T_{\Omega, \mu}\left(f_{j}\right) \chi_{k}\right|}{\eta_{1}}\right)^{q_{2}(\cdot)} \|_{\frac{p_{2}(\cdot)}{q_{2}(\cdot)}}>1
\end{array}\right.
$$

By the Proposition 3.2, we get

$$
\sum_{k=-\infty}^{\infty}\left\|\left(\frac{2^{k \alpha}\left|\sum_{j=k-1}^{k+1} T_{\Omega, \mu}\left(f_{j}\right) \chi_{k}\right|}{\eta_{1}}\right)^{q_{2}(\cdot)}\right\|_{\frac{p_{2}(\cdot)}{q_{2}(\cdot)}} \leq C \sum_{k=-\infty}^{\infty} \sum_{j=k-1}^{k+1}\left\|\frac{2^{k \alpha}\left|f_{j}\right|}{\eta_{1}}\right\|_{L^{p_{1}(\cdot)}}^{\left(q_{2}^{1}\right) k}
$$

Since $f \in \dot{K}_{p_{1}(\cdot)}^{\alpha, q_{1}(\cdot)}\left(\mathbb{R}^{n}\right)$, then we have $\left\|\frac{2^{j \alpha}\left|f \chi_{j}\right|}{\eta_{1}}\right\|_{L^{p(\cdot)}} \leq 1$, and

$$
\sum_{j=-\infty}^{\infty}\left\|\left(\frac{2^{j \alpha}\left|f \chi_{j}\right|}{\eta_{1}}\right)^{q_{1}(\cdot)}\right\|_{L_{L_{1}(\cdot)}^{q_{1}(\cdot)}} \leq 1
$$

By Lemma 3.7 and Remark 2.2, we get

$$
\begin{aligned}
& \sum_{k=-\infty}^{\infty} \|\left(\left.\frac{2^{k \alpha}\left|\sum_{j=k-1}^{k+1} T_{\Omega, \mu}\left(f_{j}\right) \chi_{k}\right|}{\eta_{1}}\right|^{q_{2}(\cdot)}\left\|_{\frac{p_{2}(\cdot)}{q_{2}(\cdot)}} \leq C \sum_{k=-\infty}^{\infty}\right\|\left(\frac{2^{k \alpha}\left|f \chi_{k}\right|}{\eta_{1}}\right)^{q_{1}(\cdot)} \|_{\frac{\left(q_{2}^{1}\right) k}{\left(q_{1}\right)_{+}}}\right. \\
& \leq C\left[\sum_{k=-\infty}^{\infty}\left\|\left(\frac{2^{k \alpha}\left|f \chi_{k}\right|}{\eta_{1}}\right)^{q_{1}(\cdot) \|}\right\|_{\frac{L^{p_{1}(\cdot)}}{L^{q_{1}(\cdot)}}}\right]^{q_{*}^{*}} \leq C
\end{aligned}
$$

Hence $\left(p_{1}\right)_{+},\left(p_{2}\right)_{-} \leq\left(q_{2}^{1}\right) k$, and $q_{*}=\min _{k \in \mathbb{N}} \frac{\left(q_{2}^{1}\right) k}{\left(q_{1}\right)_{+}}$, this implies that

$$
\eta_{12} \leq C \eta_{1} \leq C\|f\|_{\dot{K}_{p_{1}(\cdot)}^{\alpha, q_{1}(\cdot)}\left(\mathbb{R}^{n}\right)}
$$

Now, we estimate of $\eta_{11}$ using size condition of $f_{j}$ and Minkowski inequality, when $j \leq k-1$ we get,

$$
\left\|T_{\Omega, \mu}\left(f_{j}\right) \chi_{k}\right\|_{L^{p^{2}(\cdot)}\left(\mathbb{R}^{n}\right)} \leq \int_{B_{j}} f_{j}(y)\left\|\frac{\Omega(x, x-y)}{|x-y|^{n-\mu}}-\frac{\Omega(x, x)}{|x|^{n-\mu}} \mid \chi_{k}\right\|_{L^{p_{2}(\cdot)}\left(\mathbb{R}^{n}\right)} \mathrm{d} y
$$


A. Abdalmonem et al.

Since $r>p_{2}^{+}$we define the variable exponent $\frac{1}{p_{2}(x)}=\frac{1}{r}+\frac{1}{\tilde{p}_{2}(x)}$, by Lemma 3.3 we get

$$
\begin{aligned}
\left\|\mid \frac{\Omega(x, x-y)}{|x-y|^{n-\mu}}-\frac{\Omega(x, x)}{|x|^{n-\mu}} \chi_{k}\right\|_{L^{p_{2}(\cdot)}\left(\mathbb{R}^{n}\right)} & \leq\left\|\frac{\Omega(x, x-y)}{|x-y|^{n-\mu}}-\frac{\Omega(x, x)}{|x|^{n-\mu}}\right\|_{L^{r}\left(\mathbb{R}^{n}\right)}\left\|\chi_{k}\right\|_{L^{\tilde{p}_{2}(x)}\left(\mathbb{R}^{n}\right)} \\
& \leq\left\|\frac{\Omega(x, x-y)}{|x-y|^{n-\mu}}-\frac{\Omega(x, x)}{|x|^{n-\mu}}\right\|_{L^{r}\left(\mathbb{R}^{n}\right)}\left\|\chi_{B_{k}}\right\|_{L^{\tilde{p}_{2}(x)}\left(\mathbb{R}^{n}\right)}
\end{aligned}
$$

According Lemma 3.4 and the formula $\frac{1}{\tilde{p}_{2}(x)}=\frac{1}{p_{2}(x)}-\frac{1}{r}$, then we have

$$
\left\|\chi_{B_{k}}\right\|_{L^{\tilde{p}_{2}(x)}\left(\mathbb{R}^{n}\right)} \approx\left\|\chi_{B_{k}}\right\|_{L^{p_{2}(x)}\left(\mathbb{R}^{n}\right)}\left|B_{k}\right|^{\frac{-1}{r}} \approx\left\|\chi_{B_{k}}\right\|_{L^{p_{1}(x)}\left(\mathbb{R}^{n}\right)}\left|B_{k}\right|^{\frac{-1}{r}-\frac{\mu}{n}}
$$

By Lemma 3.2, we get

$$
\begin{aligned}
\left\|\frac{\Omega(x, x-y)}{|x-y|^{n-\mu}}-\frac{\Omega(x, x)}{|x|^{n-\mu}}\right\|_{L^{r}\left(\mathbb{R}^{n}\right)} & \leq C R^{\left(\frac{n}{r}-n+\mu\right)}\left(\frac{|y|}{2^{k-1}}+\int_{|y| / 2^{k}}^{|y| / 2^{k-1}} \frac{\omega_{r}(\delta)}{\delta} \mathrm{d} \delta\right) \\
& \leq C R^{\left(\frac{n}{r}-n+\mu\right)} 2^{(j-k) \beta}\left(1+\int_{0}^{1} \frac{\omega_{r}(\delta)}{\delta} \mathrm{d} \delta\right) \\
& \leq C 2^{(k-1)\left(\frac{n}{r}-n+\mu\right)}
\end{aligned}
$$

It follows that

$$
\left\|T_{\Omega, \mu}\left(f_{j}\right) \chi_{k}\right\|_{L^{p_{2}(\cdot)}\left(\mathbb{R}^{n}\right)} \leq C 2^{-k n} \int_{B_{j}} f_{j}(y) \mathrm{d} y\left\|\chi_{B_{k}}\right\|_{L^{p_{1}(x)}\left(\mathbb{R}^{n}\right)}
$$

By the Equation (1.3) and using Lemmas 3.1, 3.5, 3.6, 3.7, we can obtain

$$
\begin{aligned}
& \sum_{k=-\infty}^{\infty} \|\left(\left.\frac{2^{k \alpha}\left|\sum_{j=-\infty}^{k-2} T_{\Omega, \mu}\left(f_{j}\right) \chi_{k}\right|}{\eta_{1}}\right|^{q_{2}(\cdot)}\left\|_{\frac{p_{2}(\cdot)}{q_{2}(\cdot)}} \leq C \sum_{k=-\infty}^{\infty}\right\| \frac{2^{k \alpha}\left|\sum_{j=-\infty}^{k-2} T_{\Omega, \mu}\left(f_{j}\right) \chi_{k}\right|}{\eta_{1}}\|\|_{L^{p_{2}(\cdot)}}^{\mid\left(q_{2}^{2}\right)^{k}}\right. \\
& \leq C \sum_{k=-\infty}^{\infty}\left[2^{k \alpha} \sum_{j=-\infty}^{k-2} 2^{-k n}\left\|\frac{f_{j}}{\eta_{1}}\right\|_{L^{1}\left(\mathbb{R}^{n}\right)}\left\|\chi_{k}\right\|_{L^{p_{1}(\cdot)}}\right]^{\left(q_{2}^{2}\right) k} \leq C \sum_{k=-\infty}^{\infty}\left[2^{k \alpha} \sum_{j=-\infty}^{k-2}\left\|\frac{f_{j}}{\eta_{1}}\right\|_{L^{p_{1}(\cdot)}\left(\mathbb{R}^{n}\right)}\left\|\chi_{B_{j}}\right\|_{L^{p_{1}(\cdot)}} 2^{-k n}\left\|\chi_{B_{k}}\right\|_{L^{p_{1}(\cdot)}}\right]^{\left(q_{2}^{2}\right)^{k}} \\
& \leq C \sum_{k=-\infty}^{\infty}\left[2^{k \alpha} \sum_{j=-\infty}^{k-2}\left\|\frac{f_{j}}{\eta_{1}}\right\|_{L^{p_{1}(\cdot)}\left(\mathbb{R}^{n}\right)} \frac{\left\|\chi_{B_{j}}\right\|_{L^{p_{1}(\cdot)}}}{\left\|\chi_{B_{k}}\right\|_{L^{p^{1}(\cdot)}}}\right]^{\left(q_{2}^{2}\right)^{k}} \leq C \sum_{k=-\infty}^{\infty}\left[2^{k \alpha} \sum_{j=-\infty}^{k-2} 2^{(j-k) n \delta_{1}}\left\|\frac{f_{j}}{\eta_{1}}\right\|_{L^{p_{1}(\cdot)}\left(\mathbb{R}^{n}\right)}\right]^{\left(q_{2}^{2}\right) k} \\
& \left.\leq C \sum_{k=-\infty}^{\infty}\left[\sum_{j=-\infty}^{k-2} 2^{(j-k)\left(n \delta_{1}-\alpha\right)}\left\|\left(\frac{\left|2^{\alpha j} f_{j} \chi_{k}\right|}{\eta_{1}}\right)^{q_{1}(\cdot)}\right\|_{L^{p_{1}} \cdot(\cdot) q_{1}(\cdot)}\right]_{\left.\mathbb{R}^{n}\right)}^{\frac{1}{\left(q_{1}\right)_{+}}}\right]^{\left(q_{2}^{2}\right) k}
\end{aligned}
$$

where

1173 


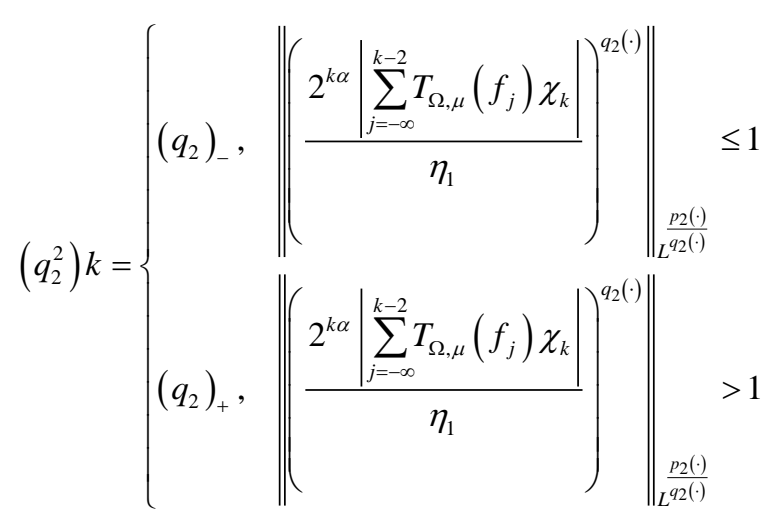

Since $f \in \dot{K}_{p_{1}(\cdot)}^{\alpha, q_{1}(\cdot)}\left(\mathbb{R}^{n}\right)$, then we have $\left\|\frac{2^{j \alpha}\left|f \chi_{j}\right|}{\eta_{1}}\right\|_{L^{p(\cdot)}} \leq 1$, and

$$
\sum_{j=-\infty}^{\infty}\left\|\left(\frac{2^{j \alpha}\left|f \chi_{j}\right|}{\eta_{1}}\right)^{q_{1}(\cdot)}\right\|_{\frac{p_{1}(\cdot)}{q_{1} \cdot(\cdot)}} \leq 1
$$

Now if $\left(q_{1}\right)_{+}<1$, then we have

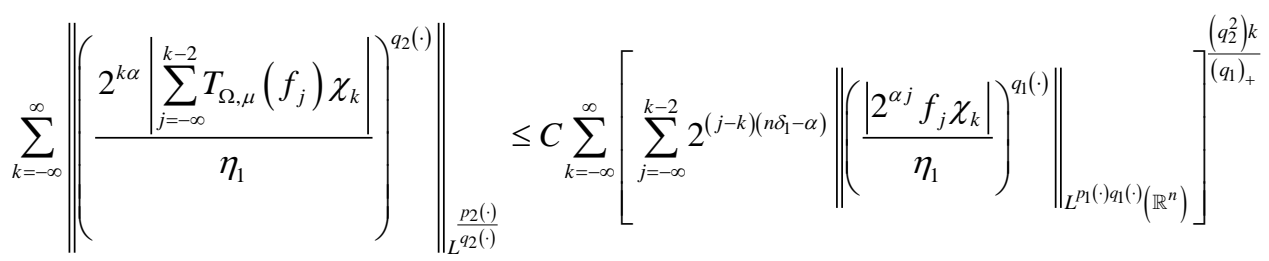

$$
\begin{aligned}
& \leq C\left[\sum_{j=-\infty}^{\infty}\left\|\left(\frac{\left|2^{\alpha j} f_{j} \chi_{k}\right|}{\eta_{1}}\right)^{q_{1}(\cdot)}\right\|_{L^{p_{1}(\cdot) q_{1}(\cdot)\left(\mathbb{R}^{n}\right)}} \sum_{k=j+2}^{\infty} 2^{(j-k)\left(n \delta_{1}-\alpha\right)}\right]^{q_{*}} \leq C
\end{aligned}
$$

where $q_{*}=\min _{k \in \mathbb{N}} \frac{\left(q_{2}^{2}\right) k}{\left(q_{1}\right)_{+}}$

If $\left(q_{1}\right)_{+} \geq 1$, then we have

$$
\begin{aligned}
& \left.\sum_{k=-\infty}^{\infty} \| \frac{2^{k \alpha}\left|\sum_{j=-\infty}^{k-2} T_{\Omega, \mu}\left(f_{j}\right) \chi_{k}\right|}{\eta_{1}}\right)^{q_{2}(\cdot)} \|_{L_{L}^{\frac{p 2}{q_{2}(\cdot)}}}
\end{aligned}
$$

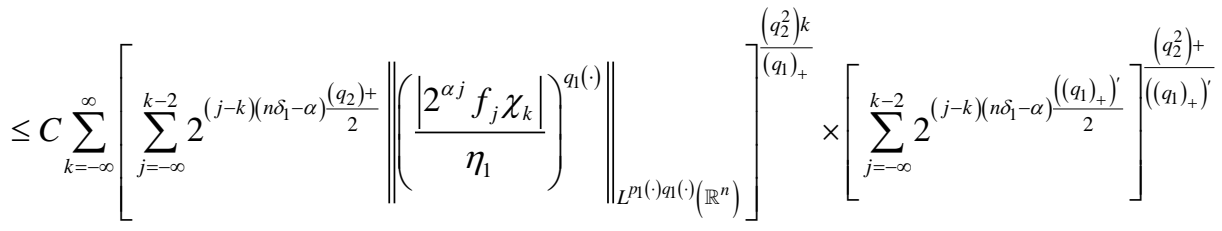

$$
\begin{aligned}
& \leq C\left[\sum_{j=-\infty}^{\infty}\left\|\left(\frac{\left|2^{\alpha j} f_{j} \chi_{k}\right|}{\eta_{1}}\right)^{q_{1}(\cdot)}\right\|_{L^{p_{1}(\cdot) q_{1}(\cdot)\left(\mathbb{R}^{n}\right)}} \sum_{k=j+2}^{\infty} 2^{(j-k)\left(n \delta_{1}-\alpha\right) \frac{\left(q_{1}\right)_{+}}{2}}\right]^{q_{*}} \leq C
\end{aligned}
$$


where $q_{*}=\min _{k \in \mathbb{N}} \frac{\left(q_{2}^{2}\right) k}{\left(q_{1}\right)_{+}}$, this implies that

$$
\eta_{11} \leq C \eta_{1} \leq C\|f\|_{\dot{K}_{p 1 \cdot(\cdot)}^{\alpha, q_{1}(\cdot)}\left(\mathbb{R}^{n}\right)}
$$

Finally, we estimate $\eta_{13}$ by Lemma 3.7, we get

$$
\begin{aligned}
& \sum_{k=-\infty}^{\infty} \|\left(\frac{\left.2^{k \alpha}\left|\sum_{j=k+2}^{\infty} T_{\Omega, \mu}\left(f_{j}\right) \chi_{k}\right|\right|^{q_{2}(\cdot)}}{\eta_{1}} \leq C \sum_{k=-\infty}^{\infty}\left\|\frac{2^{k \alpha} \mid \sum_{j=k+2}^{\infty} T_{\Omega, \mu}\left(f_{j}\right) \chi_{k} \|}{\eta_{1}}\right\| \|_{L^{p_{2}(\cdot) \cdot}}^{\left(q_{2}^{3}\right)^{k}}\right. \\
& \leq \sum_{k=-\infty}^{\infty}\left[2^{k \alpha} \sum_{j=k+2}^{\infty}\left\|\frac{1}{\eta_{1}(\cdot)} \mid \int_{C_{j}} \frac{\Omega(x, x-y)}{|x-y|^{n-\mu}} f_{j}(y) \mathrm{d} y \chi_{k}\right\|_{L_{p_{2}(\cdot)}}\right]^{\left(q_{2}^{3}\right)^{k}}
\end{aligned}
$$

Note that, when $x \in C_{k}, j \geq k+2$, then $|x-y| \sim|x|$. Therefore, applying the generalized Hölder's Inequality, we have

$$
\int_{C_{j}}\left|\frac{\Omega(x, x-y)}{|x-y|^{n-\mu}} f_{j}(y)\right| \mathrm{d} y \leq\left\|f_{j}\right\|_{L^{p_{1}(\cdot)}}\left\|\frac{\Omega(x, x-y)}{|x-y|^{n-\mu}} \chi_{j}\right\|_{L^{p^{\prime}(\cdot)}}
$$

Define the variable exponent $\frac{1}{p_{1}^{\prime}(\cdot)}=\frac{1}{r}+\frac{1}{\widetilde{p}_{1}^{\prime}(\cdot)}$ by Lemma 3.3, then we have

$$
\begin{aligned}
& \int_{C_{j}}\left|\frac{\Omega(x, x-y)}{|x-y|^{n-\mu}} f_{j}(y)\right| \mathrm{d} y \leq\left\|f_{j}\right\|_{L^{p_{1}(\cdot)}}\|\Omega(x, x-y)\|_{L^{r}}\left\|\frac{\chi_{j}}{|x-y|^{n-\mu}}\right\|_{L^{\widetilde{p}_{1}(\cdot)}} \\
& \int_{C_{j}}\left|\frac{\Omega(x, x-y)}{|x-y|^{n-\mu}} f_{j}(y)\right| d y \\
& \leq C 2^{-j(n-\mu)}\left\|f_{j}\right\|_{L^{p_{1}(\cdot)}}\left\|\chi_{j}\right\|_{L^{P_{1}(\cdot)}}\left[\int_{2^{j-2}}^{2^{j}} r^{n-1} \mathrm{~d} r\left(\int_{s^{n-1}}\left|\Omega\left(x, y^{\prime}\right)\right|^{r} \mathrm{~d} \sigma\left(y^{\prime}\right)\right)\right]^{\frac{1}{r}} \\
& \leq C 2^{-j(n-\mu)}\left\|f_{j}\right\|_{L^{\left.p^{1}()\right)}}\left\|\chi_{j}\right\|_{L^{\left.\tilde{P}^{(}()\right)}} 2^{\frac{j n}{r}}\|\Omega\|_{L^{\infty}\left(\mathbb{R}^{n}\right) \times L^{r}\left(s^{n-1}\right)} \\
& \leq C 2^{-j(n-\mu)}\left\|f_{j}\right\|_{L^{p_{1}(\cdot)}}\left\|\chi_{j}\right\|_{L^{p_{1}(\cdot)}} 2^{\frac{j n}{r}}
\end{aligned}
$$

According Lemma 3.4 and the formula $\frac{1}{\widetilde{p}_{1}^{\prime}(\cdot)}=\frac{1}{p_{1}^{\prime}(\cdot)}-\frac{1}{r}$, we have $\left\|\chi_{B_{j}}\right\|_{L^{p^{\prime}(\cdot)}} \approx\left\|\chi_{B_{j}}\right\|_{L^{p^{\prime}(\cdot)}}\left|B_{j}\right|^{\frac{-1}{r}}$ Then we get

$$
\int_{C_{j}}\left|\frac{\Omega(x, x-y)}{|x-y|^{n-\mu}} f_{j}(y)\right| \mathrm{d} y \leq 2^{-j(n-\mu)}\left\|f_{j}\right\|_{L^{p_{1}(\cdot)}}\left\|\chi_{B_{j}}\right\|_{L^{p^{i}(\cdot)}}
$$

From Equations (1.4), (1.5) and using Lemma 3.7, and $\left\|\left(\frac{2^{j \alpha}\left|f \chi_{j}\right|}{\eta_{1}}\right)^{q_{1}(\cdot)}\right\|_{\frac{p_{1}(\cdot)}{L_{11}(\cdot)}} \leq 1$ we can obtain 
A. Abdalmonem et al.

$$
\begin{aligned}
& \sum_{k=-\infty}^{\infty}\left\|\left(\frac{2^{k \alpha}\left|\sum_{j=k+2}^{\infty} T_{\Omega, \mu}\left(f_{j}\right) \chi_{k}\right|}{\eta_{1}}\right)^{q_{2}(\cdot)}\right\|_{\frac{p_{2}(\cdot)}{L^{q_{2}(\cdot)}}} \\
& \leq C \sum_{k=-\infty}^{\infty}\left[2^{k \alpha} \sum_{j=k+2}^{\infty} 2^{-j(n-\mu)}\left\|\frac{f_{j}}{\eta_{1}}\right\|_{L^{p^{1}(\cdot)}\left(\mathbb{R}^{n}\right)}\left\|\chi_{B_{j}}\right\|_{L^{\left.p_{1}^{(}()\right)}}\left\|\chi_{B_{k}}\right\|_{L^{p_{2}(\cdot)}}\right]^{\left(q_{q_{2}^{3}}^{3}\right)^{k}}
\end{aligned}
$$

Note that

$$
\left\|\chi_{B_{k}}\right\|_{L^{p_{2}(\cdot)}} \leq C 2^{-k \mu}\left\|\chi_{B_{k}}\right\|_{L^{p_{1}(\cdot)}} \quad \text { see [9]. }
$$

Then we have

$$
\begin{aligned}
& \sum_{k=-\infty}^{\infty}\left\|\left(\frac{2^{k \alpha}\left|\sum_{j=k+2}^{\infty} T_{\Omega, \mu}\left(f_{j}\right) \chi_{k}\right|}{\eta_{1}}\right)^{q_{2}(\cdot)}\right\|_{L^{p_{2}(\cdot)}} \\
& \leq C \sum_{k=-\infty}^{\infty}\left[2^{k \alpha} \sum_{j=k+2}^{\infty} 2^{j \mu}\left\|\frac{f_{j}}{\eta_{1}}\right\|_{L^{p_{1}(\cdot)}\left(\mathbb{R}^{n}\right)} 2^{-j n}\left\|\chi_{B_{j}}\right\|_{L^{p^{1}(\cdot)}} 2^{-k \mu}\left\|\chi_{B_{k}}\right\|_{L^{p_{1}(\cdot)}}\right]^{\left(q_{2}^{3}\right)^{k}} \\
& \leq C \sum_{k=-\infty}^{\infty}\left[2^{k \alpha} \sum_{j=k+2}^{\infty} 2^{(j-k) \mu}\left\|\frac{f_{j}}{\eta_{1}}\right\|_{L^{p_{1}(\cdot)}\left(\mathbb{R}^{n}\right)} \frac{\left\|\chi_{B_{k}}\right\|_{L^{p_{1}(\cdot)}}}{\left\|\chi_{B_{j}}\right\|_{L^{p_{1} \cdot(\cdot)}}}\right]^{\left(q_{2}^{3}\right) k} \\
& \leq C \sum_{k=-\infty}^{\infty}\left[2^{k \alpha} \sum_{j=k+2}^{\infty} 2^{(k-j)\left(n \delta_{2}-\mu\right)}\left\|\frac{f_{j}}{\eta_{1}}\right\|_{L^{p_{1}(\cdot)}\left(\mathbb{R}^{n}\right)}\right]^{\left(q_{2}^{3}\right) k} \\
& \leq C \sum_{k=-\infty}^{\infty}\left[\sum_{j=k+2}^{\infty} 2^{(k-j)\left(n \delta_{2}-\mu+\alpha\right)} \|\left(\frac{\left.\left|2^{\alpha j} f_{j} \chi_{k}\right|\right)^{q_{1}(\cdot)}}{\eta_{1}} \|^{\frac{1}{\left(q_{1}\right)_{+}}}\right]_{L^{p_{1}(\cdot) q_{1}(\cdot)}\left(\mathbb{R}^{n}\right)}^{\left(q_{2}^{3}\right) k}\right.
\end{aligned}
$$

where

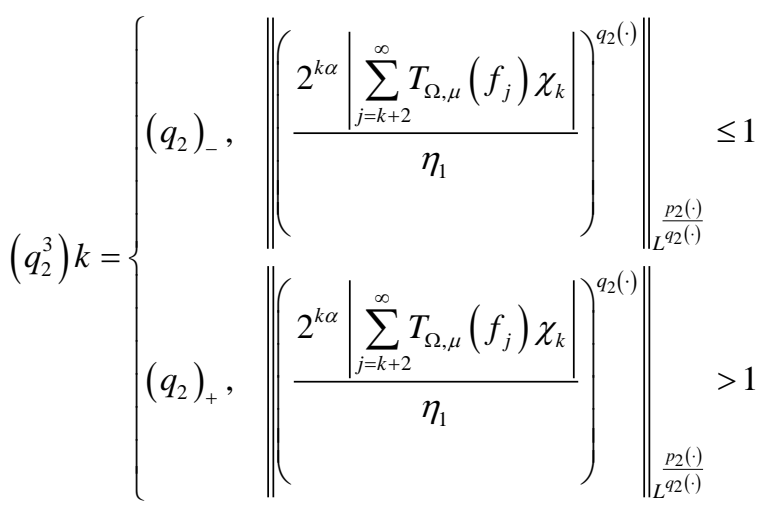

Since $\left(q_{2}\right)_{-} \geq\left(q_{1}\right)_{+}$and $\alpha>\mu-n \delta_{2}$, as the same $\eta_{11}$ we have

1176 


$$
\eta_{13} \leq C \eta_{1} \leq C\|f\|_{\dot{K}_{p_{1}(\cdot)}^{\alpha, q_{1} \cdot(\cdot)}\left(\mathbb{R}^{n}\right)}
$$

This completes the proof Theorem 1.

\section{Proof of Theorem 2}

Let $b \in \operatorname{Lip}_{\beta}\left(\mathbb{R}^{n}\right), \quad f \in \dot{K}_{p_{1}(\cdot)}^{\left.\alpha, q_{1} \cdot \cdot\right)}\left(\mathbb{R}^{n}\right)$. We write

$$
f(x)=\sum_{j=-\infty}^{\infty} f(x) \chi_{k}=\sum_{j=-\infty}^{\infty} f_{j}(x)
$$

From definition of $\dot{K}_{p(\cdot)}^{\alpha, q(\cdot)}\left(\mathbb{R}^{n}\right)$

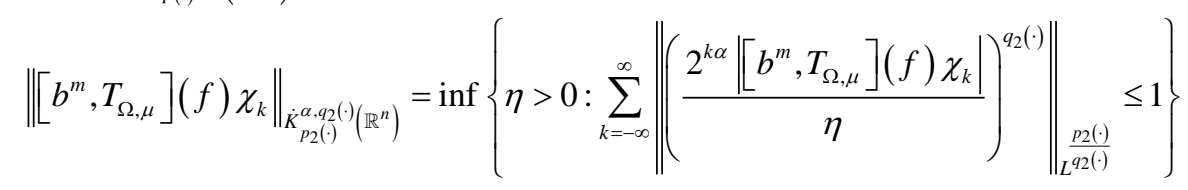

Since

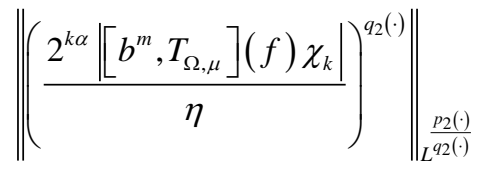

$$
\begin{aligned}
& \left.\left.\leq \| \frac{2^{k \alpha}\left|\sum_{j=-\infty}^{\infty}\left[b^{m}, T_{\Omega, \mu}\right](f) \chi_{k}\right|}{\eta_{21}+\eta_{22}+\eta_{23}}\right)^{q_{2}(\cdot)}\left\|_{\frac{p_{2}(\cdot)}{q_{2}(\cdot)}} \leq\right\| \frac{2^{k \alpha}\left|\sum_{j=-\infty}^{k-2}\left[b^{m}, T_{\Omega, \mu}\right](f) \chi_{k}\right|}{\eta_{21}}\right)^{q_{2}(\cdot)} \|_{L_{L^{2}}}^{p_{2(\cdot)}(\cdot)}
\end{aligned}
$$

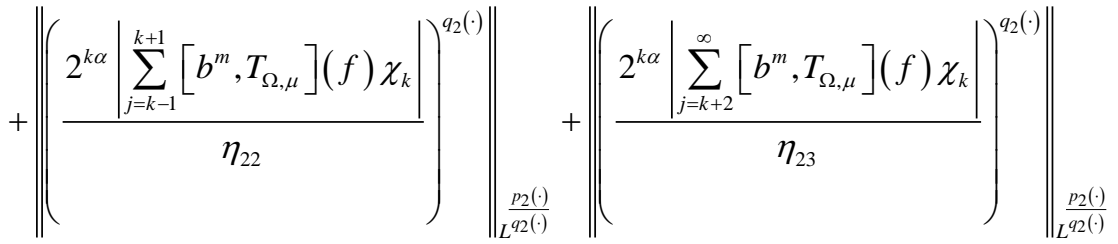

where

$$
\begin{aligned}
& \eta_{21}=\left\|\left\{2^{k \alpha}\left|\sum_{j=-\infty}^{k-2}\left[b^{m}, T_{\Omega, \mu}\right](f) \chi_{k}\right|\right\}_{k=-\infty}^{\infty}\right\|_{I^{22}(\cdot)\left(L^{p_{2}(\cdot)}\right)} \\
& \eta_{22}=\left\|\left\{2^{k \alpha}\left|\sum_{j=k-1}^{k+1}\left[b^{m}, T_{\Omega, \mu}\right](f) \chi_{k}\right|\right\}_{k=-\infty}^{\infty}\right\| \|_{I_{2}(\cdot)\left(L^{p_{2}(\cdot)}\right)} \\
& \eta_{23}=\left\|\left\{2^{k \alpha}\left|\sum_{j=k+2}^{\infty}\left[b^{m}, T_{\Omega, \mu}\right](f) \chi_{k}\right|\right\}_{k=-\infty}^{\infty}\right\| \|_{g_{2}(\cdot)\left(L^{p^{2}(\cdot)}\right)}
\end{aligned}
$$

And $\eta=\eta_{21}+\eta_{22}+\eta_{23}$. The similar to prove of Theorem 1

$$
\left\|\left[b^{m}, T_{\Omega, \mu}\right](f) \chi_{k}\right\|_{\dot{K}_{p_{2}(\cdot)}^{\alpha, q_{2}(\cdot)}\left(\mathbb{R}^{n}\right)} \leq C \eta \leq C\left[\eta_{21}+\eta_{22}+\eta_{23}\right]
$$

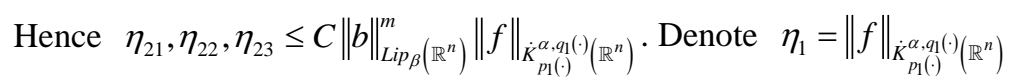

First we estimate $\eta_{22}$. Note that $\left[b^{m}, T_{\Omega, \mu}\right]$ is bonuded on $L^{p(\cdot)}\left(\mathbb{R}^{n}\right)$ (Proposition 3.3), similarly to esti- 
mate for $\eta_{12}$ in the proof of the Theorem 1, we get that

$$
\sum_{k=-\infty}^{\infty} \|\left.\left(\frac{2^{k \alpha}\left|\sum_{j=k-1}^{k+1}\left[b^{m}, T_{\Omega, \mu}\right]\left(f_{j}\right) \chi_{k}\right|}{\eta_{1}}\right)^{q_{2}(\cdot)}\right|_{\substack{\frac{p_{2}(\cdot)}{q_{2}(\cdot)}}} \leq C
$$

That is

$$
\eta_{22} \leq C \eta_{1}\|b\|_{L i p_{\beta}\left(\mathbb{R}^{n}\right)}^{m} \leq C\|b\|_{L_{\beta}\left(\mathbb{R}^{n}\right)}^{m}\|f\|_{\dot{K}_{p_{1}(\cdot)}^{\alpha, q_{1}(\cdot)}\left(\mathbb{R}^{n}\right)}
$$

Now, we estimate of $\eta_{21}$. Using size condition of $f_{j}$ and Minkowski inequality, when $j \leq k-1$ we get,

$$
\begin{aligned}
\left|\left[b^{m}, T_{\Omega, \mu}\right]\right| & =\left|\int_{\mathbb{R}^{n}} \frac{\Omega(x, x-y)}{|x-y|^{n-\mu}}(b(x)-b(y))^{m} f(y) \mathrm{d} y\right| \\
& \leq\left|\int_{\mathbb{R}^{n}} \frac{\Omega(x, x-y)}{|x-y|^{n-\mu}}\right| x-\left.y\right|^{m \beta} \|\left. b\right|_{L_{i p_{\beta}}} ^{m} f(y) \mathrm{d} y \mid \\
& \leq\|b\|_{L_{i p_{\beta}}}^{m} \int_{\mathbb{R}^{n}} \frac{|\Omega(x, x-y)|}{|x-y|^{n-\mu n-\mu-m \beta}}|f(y)| \mathrm{d} y \\
& \leq\|b\|_{L_{i p_{\beta}}}^{m} \int_{\mathbb{R}^{n}} \frac{|\Omega(x, x-y)|}{|x-y|^{n-\mu n-(\mu+m \beta)}} f(y) \mathrm{d} y
\end{aligned}
$$

We have that

$$
\left\|\left[b^{m}, T_{\Omega, \mu}\right] \chi_{k}\right\|_{L^{p_{2}(\cdot)}} \leq C\|b\|_{L_{i p_{\beta}}}^{m} \int_{B_{j}} f_{j}(y)\left\|\frac{\Omega(x, x-y)}{|x-y|^{n-(\mu+m \beta)}}-\frac{\Omega(x, x)}{|x|^{n-(\mu+m \beta)}} \mid \chi_{k}\right\|_{L^{p^{2} \cdot(\cdot)}\left(\mathbb{R}^{n}\right)} \mathrm{d} y
$$

The similar way to estimate of $T_{\Omega, \mu}$ in the proof of Theorem 1, we get that

$$
\left\|\left[b^{m}, T_{\Omega, \mu}\right] \chi_{k}\right\|_{L^{p_{2}(\cdot)}} \leq C\|b\|_{L_{i p_{\beta}}}^{m} 2^{-k n} \int_{B_{j}} f_{j}(y) \mathrm{d} y\left\|\chi_{B_{k}}\right\|_{L^{p_{1}(x)}\left(\mathbb{R}^{n}\right)}
$$

By (1.7) and lemma 3.7, we obtain that

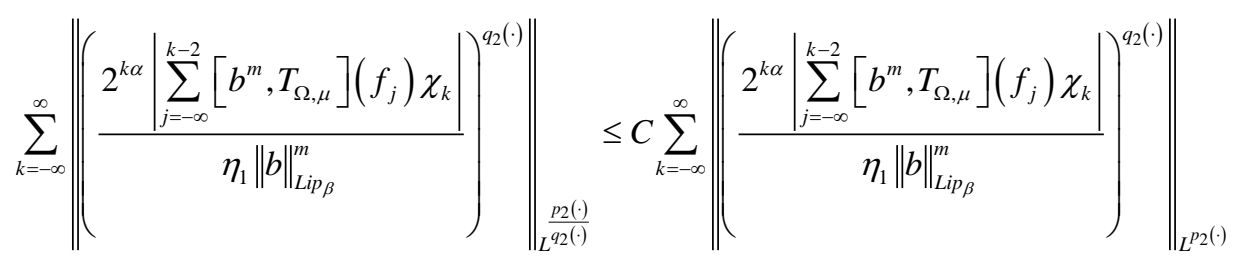

$$
\begin{aligned}
& \leq C \sum_{k=-\infty}^{\infty}\left[2^{k \alpha} \sum_{j=-\infty}^{k-2} 2^{-k n}\left\|\frac{f_{j}}{\eta_{1}}\right\|_{L^{1}\left(\mathbb{R}^{n}\right)}\left\|\chi_{k}\right\|_{L^{p_{1}(\cdot)}}\right]^{\left(q_{2}^{2}\right)^{k}} \leq C \sum_{k=-\infty}^{\infty}\left[2^{k \alpha} \sum_{j=-\infty}^{k-2}\left\|\frac{f_{j}}{\eta_{1}}\right\|_{L^{p^{1}(\cdot)}\left(\mathbb{R}^{n}\right)}\left\|\chi_{B_{j}}\right\|_{L^{p_{1}^{(} \cdot()}} 2^{-k n}\left\|\chi_{B_{k}}\right\|_{L^{p_{1}(\cdot)}}\right]^{\left(q_{2}^{2}\right)^{k}}
\end{aligned}
$$

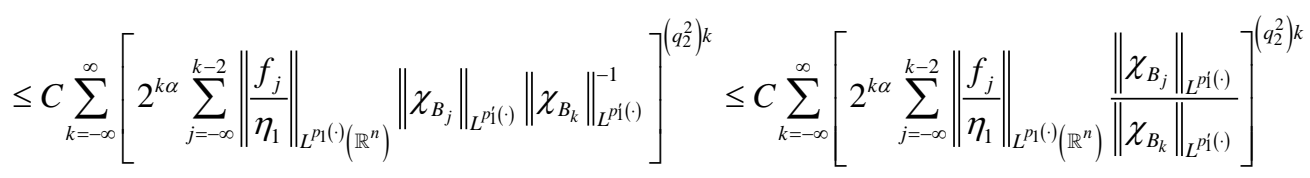

$$
\begin{aligned}
& \leq C \sum_{k=-\infty}^{\infty}\left[2^{k \alpha} \sum_{j=-\infty}^{k-2} 2^{(j-k) n \delta_{1}}\left\|\frac{f_{j}}{\eta_{1}}\right\|_{L^{p_{1}(\cdot)}\left(\mathbb{R}^{n}\right)}\right]^{\left(q_{2}^{2}\right)^{k}} \leq C \sum_{k=-\infty}^{\infty}\left[\sum_{j=-\infty}^{k-2} 2^{(j-k)\left(n \delta_{1}-\alpha\right)}\left\|\left(\frac{\left|2^{\alpha j} f_{j} \chi_{k}\right|}{\eta_{1}}\right)^{q_{1}(\cdot)}\right\|_{L^{p_{1}(\cdot) \cdot q_{1}(\cdot)\left(\mathbb{R}^{n}\right)}}^{\frac{1}{\left(q_{1}\right)_{+}}}\right]^{\left(q_{2}^{2}\right)^{k}}
\end{aligned}
$$


where

$$
\left(q_{2}^{2}\right) k=\left\{\begin{array}{l|l}
\left(q_{2}\right)_{-}, & \left.\| \frac{2^{k \alpha}\left|\sum_{j=\infty}^{k-2}\left[b^{m}, T_{\Omega, \mu}\right]\left(f_{j}\right) \chi_{k}\right|}{\eta_{1}\|b\|_{L i p_{\beta}}^{m}}\right)^{q_{2}(\cdot)} \|_{\frac{p_{2}(\cdot)}{q_{2}(\cdot)}} \leq 1 \\
\left(q_{2}\right)_{+}, & \left\|\left(\frac{2^{k \alpha}\left|\sum_{j=k-1}^{\infty}\left[b^{m}, T_{\Omega, \mu}\right]\left(f_{j}\right) \chi_{k}\right|}{\eta_{1}\|b\|_{L i p_{\beta}}^{m}}\right)^{q_{2}(\cdot)}\right\|_{\frac{p_{2}(\cdot)}{q_{2}(\cdot)}}>1
\end{array}\right.
$$

Since $\left(q_{2}\right)_{-} \geq\left(q_{1}\right)_{+}$and $\alpha>-n \delta_{1}$, the similar way to estimate $\eta_{11}$ in the proof of Theorem1, we can obtain that

$$
\begin{aligned}
& \left.\sum_{k=-\infty}^{\infty}\left\|\left(\frac{2^{k \alpha}\left|\sum_{j=-\infty}^{k-2}\left[b^{m}, T_{\Omega, \mu}\right]\left(f_{j}\right) \chi_{k}\right|}{\eta_{1}}\right)^{q_{2}(\cdot)}\right\|_{p_{L}^{\frac{p_{2}(\cdot)}{q_{2}(\cdot)}}}\left\|\left(\frac{\left|2^{\alpha j} f_{j} \chi_{k}\right|}{\eta_{1}}\right)^{q_{1}(\cdot)}\right\|_{k=-\infty}\right]_{L^{p_{1}(\cdot) q_{1}(\cdot)}\left(\mathbb{R}^{n}\right)} \leq C
\end{aligned}
$$

where $q_{*}=\min _{k \in \mathbb{N}} \frac{\left(q_{2}^{2}\right) k}{\left(q_{1}\right)_{+}}$, this implies that

$$
\eta_{21} \leq C \eta_{1}\|b\|_{L i p_{\beta}\left(\mathbb{R}^{n}\right)}^{m} \leq C\|b\|_{L i p_{\beta}\left(\mathbb{R}^{n}\right)}^{m}\|f\|_{\dot{K}_{p_{1}(\cdot)}^{\alpha, q_{1}(\cdot)}}\left(\mathbb{R}^{n}\right)
$$

Finally, we estimate $\eta_{23}$. Note that, when $x \in C_{k}, \quad j \geq k+2$, then $|x-y| \sim|y|$, we can obtain that

$$
\begin{aligned}
\left|\left[b^{m}, T_{\Omega, \mu}\right]\right| & =\left|\int_{\mathbb{R}^{n}} \frac{\Omega(x, x-y)}{|x-y|^{n-\mu}}(b(x)-b(y))^{m} f(y) \mathrm{d} y\right| \\
& \leq\left|\int_{\mathbb{R}^{n}} \frac{\Omega(x, x-y)}{|x-y|^{n-\mu}}\right| x-\left.y\right|^{m \beta}\|b\|_{L i p_{\beta}}^{m} f(y) \mathrm{d} y \mid \\
& \leq \|\left. b\right|_{L i p_{\beta}} ^{m} \int_{\mathbb{R}^{n}} \frac{|\Omega(x, x-y)|}{|x-y|^{n-(\mu+m \beta)}} f(y) \mathrm{d} y
\end{aligned}
$$

Then we have

$$
\left|\left[b^{m}, T_{\Omega, \mu}\right]\right| \leq\|b\|_{L i p_{\beta}}^{m} \int_{\mathbb{R}^{n}} \frac{|\Omega(x, x-y)|}{|x-y|^{n-(\mu+m \beta)}} f(y) \mathrm{d} y
$$

Applying the generalized Hölder’s Inequality, we get

$$
\int_{C_{j}}\left|\frac{|\Omega(x, x-y)|}{|x-y|^{n-(\mu+m \beta)}} f_{j}(y)\right| \mathrm{d} y \leq\left\|f_{j}\right\|_{L^{p_{1}(\cdot)}}\left\|\frac{\Omega(x, x-y)}{|x-y|^{n-(\mu+m \beta)}} \chi_{j}\right\|_{L^{p^{i}(\cdot)}}
$$


A. Abdalmonem et al.

Define the variable exponent $\frac{1}{p_{1}^{\prime}(\cdot)}=\frac{1}{r}+\frac{1}{\widetilde{p_{1}^{\prime}(\cdot)}}$ by Lemma 3.3, then we have

$$
\begin{aligned}
& \int_{C_{j}}\left|\frac{\Omega(x, x-y)}{|x-y|^{n-(\mu+m \beta)}} f_{j}(y)\right| d y \\
& \leq\left\|f_{j}\right\|_{L^{p_{1}(\cdot)}}\|\Omega(x, x-y)\|_{L^{r}}\left\|\frac{\chi_{j}}{|x-y|^{n-(\mu+m \beta)}}\right\|_{L_{\left.L_{1}^{(} \cdot\right)}} \\
& \leq C 2^{-j(n-(\mu+m \beta))}\left\|f_{j}\right\|_{L^{p_{1}(\cdot)}}\left\|\chi_{j}\right\|_{L^{p_{1}^{\prime}(\cdot)}}\left[\int_{2^{j-2}}^{2^{j}} r^{n-1} \mathrm{~d} r\left(\int_{s^{n-1}}\left|\Omega\left(x, y^{\prime}\right)\right|^{r} \mathrm{~d} \sigma\left(y^{\prime}\right)\right)\right]^{\frac{1}{r}} \\
& \leq C 2^{-j(n-(\mu+m \beta))}\left\|f_{j}\right\|_{L^{p_{1}(\cdot)}}\left\|\chi_{j}\right\|_{L^{\bar{p}(\cdot)}} 2^{\frac{j n}{r}}\|\Omega\|_{L^{\infty}\left(\mathbb{R}^{n}\right) \times L^{r}\left(S^{n-1}\right)} \\
& \leq C 2^{-j(n-(\mu+m \beta))}\left\|f_{j}\right\|_{L^{p_{1}(\cdot)}}\left\|\chi_{j}\right\|_{L^{\widetilde{p}_{1}(\cdot)}} 2^{\frac{j n}{r}}
\end{aligned}
$$

According Lemma 3.4 and the formula $\frac{1}{\widetilde{p_{1}^{\prime}(\cdot)}}=\frac{1}{p_{1}^{\prime}(\cdot)}-\frac{1}{r}$, we have $\left\|\chi_{B_{j}}\right\|_{L^{p i}(\cdot)} \approx\left\|\chi_{B_{j}}\right\|_{L^{p}(\cdot)}\left|B_{j}\right|^{\frac{-1}{r}}$. Then we get

$$
\begin{aligned}
\int_{C_{j}}\left|\frac{\Omega(x, x-y)}{|x-y|^{n-(\mu+m \beta)}} f_{j}(y)\right| \mathrm{d} y & \leq 2^{-j(n-(\mu+m \beta))}\left\|f_{j}\right\|_{L^{p_{1}(\cdot)}}\left\|\chi_{B_{j}}\right\|_{L^{p_{1}(\cdot)}} 2^{\frac{j n}{r}} 2^{\frac{-j n}{r}} \\
& \leq 2^{-j(n-(\mu+m \beta))}\left\|f_{j}\right\|_{L^{p_{1}(\cdot)}}\left\|\chi_{B_{j}}\right\|_{L^{p^{\prime}(\cdot)}}
\end{aligned}
$$

By (1.8), we can obtain that

$$
\left.\| b^{m}, T_{\Omega, \mu}\right] \mid \leq C 2^{-j(n-(\mu+m \beta))}\|b\|_{L_{i p_{\beta}}}^{m}\left\|f_{j}\right\|_{L^{p_{1}(\cdot)}}\left\|\chi_{B_{j}}\right\|_{L^{p^{i}(\cdot)}}
$$

Then by (1.9) and Lemma 3.7, we have

$$
\sum_{k=-\infty}^{\infty}\left\|\left(\frac{2^{k \alpha}\left|\sum_{j=k+2}^{\infty}\left[b^{m}, T_{\Omega, \mu}\right]\left(f_{j}\right) \chi_{k}\right|}{\eta_{1}\|b\|_{L i p_{\beta}}^{m}}\right)^{q_{2}(\cdot)}\right\|_{\frac{p_{2}(\cdot)}{q_{2}(\cdot)}} \leq C \sum_{k=-\infty}^{\infty}\left\|\left(\frac{2^{k \alpha}\left|\sum_{j=k+2}^{\infty}\left[b^{m}, T_{\Omega, \mu}\right]\left(f_{j}\right) \chi_{k}\right|}{\eta_{1}\|b\|_{L i p_{\beta}}^{m}}\right)^{q_{2}(\cdot)}\right\|_{L^{p_{2}(\cdot)}}
$$

where

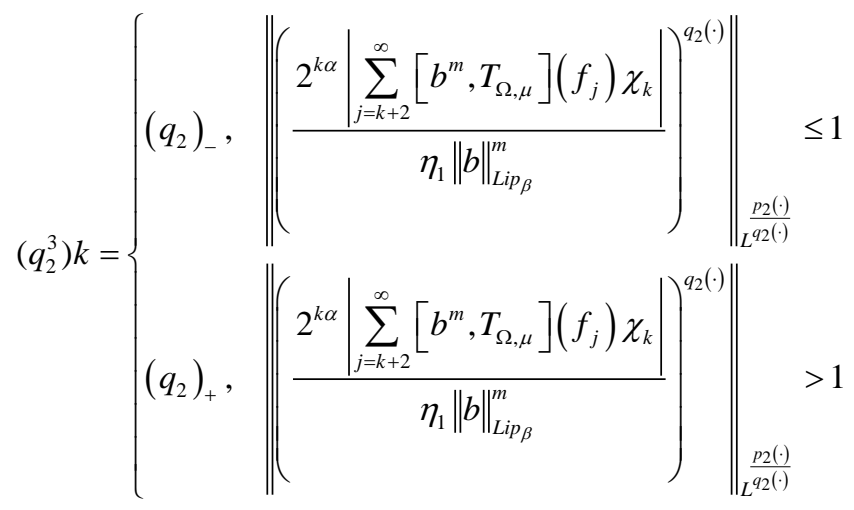

Furthermore, when $\alpha>(\mu+m \beta)-n \delta_{2}$, note that $\left\|\chi_{B_{k}}\right\|_{L^{p_{2}(\cdot)}} \leq C 2^{-k(\mu+m \beta)}\left\|\chi_{B_{k}}\right\|_{L^{p_{1}(\cdot)}}$ see [9], the similar way to estimate $\eta_{11}$, we get

1180 


$$
\begin{aligned}
& \sum_{k=-\infty}^{\infty}\left\|\left(\frac{2^{k \alpha}\left|\sum_{j=k+2}^{\infty}\left[b^{m}, T_{\Omega, \mu}\right]\left(f_{j}\right) \chi_{k}\right|}{\eta_{1}\|b\|_{L i p_{\beta}}^{m}}\right)^{q_{2}(\cdot)}\right\|_{\frac{p_{2}(\cdot)}{q_{2}(\cdot)}} \\
& \leq C \sum_{k=-\infty}^{\infty}\left[2^{k \alpha} \sum_{j=k+2}^{\infty} 2^{-j(n-(\mu+m \beta))}\left\|\frac{f_{j}}{\eta_{1}}\right\|_{L^{p_{1}(\cdot)}\left(\mathbb{R}^{n}\right)}\left\|\chi_{B_{j}}\right\|_{L^{p^{i}(\cdot)}}\left\|\chi_{B_{k}}\right\|_{L^{p_{2}(\cdot)}}\right]^{\left(q^{3}\right) k} \\
& \leq C \sum_{k=-\infty}^{\infty}\left[2^{k \alpha} \sum_{j=k+2}^{\infty} 2^{-j(n-(\mu+m \beta))}\left\|\frac{f_{j}}{\eta_{1}}\right\|_{L^{p_{1}(\cdot)}\left(\mathbb{R}^{n}\right)}\left\|\chi_{B_{j}}\right\|_{L^{p^{i}(\cdot)}} 2^{-k(\mu+m \beta)}\left\|\chi_{B_{k}}\right\|_{L^{p_{1} \cdot(\cdot)}}\right]^{\left(q_{2}^{3}\right) k} \\
& \leq C \sum_{k=-\infty}^{\infty}\left[2^{k \alpha} \sum_{j=k+2}^{\infty} 2^{(j-k)(\mu+m \beta)}\left\|\frac{f_{j}}{\eta_{1}}\right\|_{L^{p_{1} \cdot(\cdot)}\left(\mathbb{R}^{n}\right)}\left\|\chi_{B_{j}}\right\|_{L^{p_{1}(\cdot)}}^{-1}\left\|\chi_{B_{k}}\right\|_{L^{p_{1} \cdot(\cdot)}}\right]^{\left(q^{3}\right) k} \\
& \leq C \sum_{k=-\infty}^{\infty}\left[2^{k \alpha} \sum_{j=k+2}^{\infty} 2^{(j-k)(\mu+m \beta)}\left\|\frac{f_{j}}{\eta_{1}}\right\|_{L^{p_{1}(\cdot)}\left(\mathbb{R}^{n}\right)} \frac{\left\|\chi_{B_{k}}\right\|_{L^{p_{1}(\cdot)}}}{\left\|\chi_{B_{j}}\right\|_{L^{p_{1} \cdot(\cdot)}}}\right]^{\left(q_{2}^{3}\right) k} \\
& \leq C \sum_{k=-\infty}^{\infty}\left[2^{k \alpha} \sum_{j=k+2}^{\infty} 2^{(k-j)\left(n \delta_{2}-(\mu+m \beta)\right)}\left\|\frac{f_{j}}{\eta_{1}}\right\|_{L^{p_{1}(\cdot)}\left(\mathbb{R}^{n}\right)}\right]^{\left(q_{2}^{3}\right) k} \\
& \left.\left.\leq C \sum_{k=-\infty}^{\infty}\left[\sum_{j=k+2}^{\infty} 2^{(k-j)\left(n \delta_{2}-(\mu+m \beta)+\alpha\right)}\left\|\left(\frac{\left|2^{\alpha j} f_{j} \chi_{k}\right|}{\eta_{1}}\right)^{q_{1} \cdot(\cdot)}\right\|_{L_{L^{p_{1}} \cdot(\cdot) q_{1}(\cdot)}\left(\frac{1}{\left.q_{1}\right)_{+}}\right.}\right]^{n}\right)\right]^{\left(q_{2}^{3}\right) k}
\end{aligned}
$$

We can conclude that

$$
\begin{aligned}
& \sum_{k=-\infty}^{\infty}\left\|\frac{\left.2^{k \alpha}\left|\sum_{j=k+2}^{\infty}\left[b^{m}, T_{\Omega, \mu}\right]\left(f_{j}\right) \chi_{k}\right|\right|^{q_{2}(\cdot)}}{\eta_{1}\|b\|_{L_{i p_{\beta}}}^{m}}\right\|_{\frac{p_{L_{2}(\cdot)}}{q_{q_{2}(\cdot)}}} \| C \sum_{k=-\infty}^{\infty}\left[\sum_{j=k+2}^{\infty} 2^{(k-j)\left(n \delta_{2}-(\mu+m \beta)+\alpha\right)}\left\|\left(\frac{\left|2^{\alpha j} f_{j} \chi_{k}\right|}{\eta_{1}}\right)^{q_{1}(\cdot)}\right\|_{\left.L^{p_{1}(\cdot) q_{1}(\cdot)\left(\mathbb{R}^{n}\right)}\right]^{q_{*}} \leq C} \leq C\right.
\end{aligned}
$$

where $q_{*}=\min _{k \in \mathbb{N}} \frac{\left(q_{2}^{3}\right) k}{\left(q_{1}\right)_{+}}$, this implies that

$$
\eta_{23} \leq C \eta_{1}\|b\|_{L i p_{\beta}\left(\mathbb{R}^{n}\right)}^{m} \leq C\|b\|_{L i_{\beta}\left(\mathbb{R}^{n}\right)}^{m}\|f\|_{\dot{K}_{p_{1}(\cdot)}^{\alpha, q_{1}(\cdot)}\left(\mathbb{R}^{n}\right)}
$$

This completes the proof Theorem 2.

\section{Competing Interests}

The authors declare that they have no competing interests. 


\section{Acknowledgements}

This paper is supported by National Natural Foundation of China (Grant No. 11561062).

\section{References}

[1] Cruz-Uribe, D. and Fiorenza, A. (2013) Variable Lebesgue Spaces. Foundations and Harmonic Analysis. Applied and Numerical Harmonic Analysis, Springer, New York.

[2] Kenig, C. (1994) Harmonic Analysis Techniques for Second Order Elliptic Boundary Value Problems. American Mathematical Society, Providence. http://dx.doi.org/10.1090/cbms/083

[3] Calderón, A. and Zygmund, A. (1955) On a Problem of Mihilim. Transations of the American Mathematical Society, 78, 209-224. http://dx.doi.org/10.2307/1992955

[4] Calderón, A. and Zygmund, A. (1978) On Singular Integral with Variable Kernels. Journal of Applied Analysis, 7, 221-238. http://dx.doi.org/10.1080/00036817808839193

[5] Christ, M., Duoandikoetxea, J. and Rubio de Francia, J. (1986) Maximal Operators Related to the Radon Transform and the Calderóon-Zygmund Method of Rotations. Duke Mathematical Journal, 53, 189-209. http://dx.doi.org/10.1215/S0012-7094-86-05313-5

[6] Muckenhoupt, B. and Wheeden, R. (1971) Weighted Norm Inequalities for Singular and Fractional Integrals. Transations of the American Mathematical Society, 161, 249-258. http://dx.doi.org/10.1090/S0002-9947-1971-0285938-7

[7] Kováčik, O. and Rákosník, J. (1991) On Spaces $L^{p(x)}$ and $W^{k, p(x)}$. Czechoslovak Matematical Journal, 41, 592-618.

[8] Izuki, M. (2010) Boundedness of Commutators on Herz Spaces with Variable Exponent. Rendiconti del Circolo Matematico di Palermo, 59, 199-213. http://dx.doi.org/10.1007/s12215-010-0015-1

[9] Izuki, M. (2010) Fractional Integrals on Herz-Morrey Spaces with Variable Exponent. Hiroshima Mathematical Journal, 40, 343-355.

[10] Wang, L. and Tao, S. (2014) Boundedness of Littlewood-Paley Operators and Their Commutators on Herz-Morrey Spaces with Variable Exponent. Journal of Inequalities and Applications, 227, 1-17. http://dx.doi.org/10.1186/1029-242x-2014-227

[11] Wang, L. and Tao, S. (2015) Parameterized Littlewood-Paley Operators and Their Commutators on Lebegue Spaces with variable Exponent. Analysis in Theory and Applications, 31, 13-24.

[12] Izuki, M. (2009) Herz and Amalgam Spaces with Variable Exponent, the Haar Wavelets and Greediness of the Wavelet System. East Journal on Approximations, 15, 87-109.

[13] Tan, J. and Liu, Z. (2015) Some Boundedness of Homogeneous Fractional Integrals on Variable Exponent Function Spaces. ACTA Mathematics Science (Chinese Series), 58, 310-320.

[14] Afif, A., Omer, A. and Tao, S. (2016) The Boundedness of Fractional Integral with Variable Kernel on Variable Exponent Herz-Morrey Spaces. Jouranl of Applied Mathematics and Physics, 4, 187-195.

[15] Wu, H. and Lan, J. (2012) The Boundedness for a Class of Rough Fractional Integral Operators on Variable Exponent Lebesgue Spaces. Analysis in Theory and Applications, 28, 286-293.

[16] Wang, L. and Tao, S. (2016) Parameterized Littlewood-Paley Operators and Their Commutators on Herz Spaces with Variable Exponents. Turkish Journal of Mathematics, 40, 122-145. http://dx.doi.org/10.3906/mat-1412-52

[17] Almeida, A., Hasanov, J. and Samko, S. (2008) Maximal and Potential Operators in Variable Exponent Morrey Spaces. Georgian Mathematical Journal, 15, 195-208.

[18] Stien, E. (1970) Singular Integral and Differentiability Proerties of Functions. Princeton University Press, Princeton.

[19] Cruz-Uribe, D., Fiorenza, A., Martell, J. and Pérez, C. (2006) The Boundedness of Classical Operators on Variable $L^{p} \quad$ Spaces. Annales Academiae Scientiarum Fennicae Mathematica, 31, $239-264$.

[20] Diening, L., Harjulehto, P., Hästö, P. and Ružičcka, M. (2011) Lebesgue and Sobolev Spaces with Variable Exponents. Springer-Verlag Berlin Heidelberg.

[21] Ding, Y. and Lu, S. (2000) Homogeneous Fractional Integrals on Hardy Spaces. Tohoku Mathematical Journal, 52, 153-162. http://dx.doi.org/10.2748/tmj/1178224663

[22] Karlovich, A.Y. and Lerner, A.K. (2005) Commutators of Singular Integral on Generalized $L^{p}$ Spaces with Variable Exponent. Publicacions Matemàtiques, 49, 111-125. 


\section{Submit or recommend next manuscript to SCIRP and we will provide best service for you:}

Accepting pre-submission inquiries through Email, Facebook, Linkedin, Twitter, etc A wide selection of journals (inclusive of 9 subjects, more than 200 journals)

Providing a 24-hour high-quality service

User-friendly online submission system

Fair and swift peer-review system

Efficient typesetting and proofreading procedure

Display of the result of downloads and visits, as well as the number of cited articles

Maximum dissemination of your research work

Submit your manuscript at: http://papersubmission.scirp.org/ 